\title{
Metal Oxide Heteronanostructures Prepared by Electrospinning for the Humidity Detection: Fundamentals and Perspectives
}

\author{
Victor N. S. Leão, Evando S. Araújo* (1) \\ Research Group on Electrospinning and Nanotechnology Applications (GPEA-Nano), \\ Universidade Federal do Vale do São Francisco, Juazeiro, Brazil \\ Email: *evando.araujo@univasf.edu.br
}

How to cite this paper: Leão, V.N.S. and Araújo, E.S. (2019) Metal Oxide Heteronanostructures Prepared by Electrospinning for the Humidity Detection: Fundamentals and Perspectives. Journal of Materials Science and Chemical Engineering, 7, 43-54. https://doi.org/10.4236/msce.2019.77006

Received: June 17, 2019

Accepted: July 19, 2019

Published: July 22, 2019

Copyright () 2019 by author(s) and Scientific Research Publishing Inc. This work is licensed under the Creative Commons Attribution International License (CC BY 4.0).

http://creativecommons.org/licenses/by/4.0/

\begin{abstract}
Humidity is a critical environmental parameter for several production processes and its control/monitoring is of great importance in maintaining the quality of goods and products. In this context, metallic oxide ceramic nanostructures are materials of great technological interest in the fabrication of moisture sensors because they have good chemical/structural stability and high surface area/volume ratio. The electrical response of these sensors relates to the chemisorbed and physisorbed layers of water molecules on the surface of the ceramic particles and to the capillary condensation of water in the microscopic pores between the particles. Based on these aspects, this work presents the fundamentals, electrical/electronic properties, influence of dopants, novel preparation procedure by electrospinning and perspectives of application of $\mathrm{TiO}_{2}: \mathrm{WO}_{3}$ metal oxide heteronanostructures as humidity sensors.
\end{abstract}

\section{Keywords}

Metal Oxides, Heteronanostructures, Electrospinning, Humidity Sensors

\section{Introduction}

Humidity is a parameter of great importance for the better quality in the production, storage and transport of food, goods and medicines by the industry [1]. In this sense, relative humidity (RH), which is given by the ratio of actual vapor pressure to saturated vapor pressure at a given temperature, is the most frequently used parameter for measuring moisture.

Humidity sensors can be produced from polymeric or ceramic materials. In 
addition, recent works also show significant advances in the use of hybrid nanocomposites based on selective polymers, metal oxides and graphene oxide for the fabrication of moisture sensors [2] [3] [4] [5] [6]. Ceramic-based sensors generally consist of metal oxides and show superior performance to the polymer because they work in a large window of temperatures, high chemical stability and rapid response to changes in moisture [1]. Several mechanisms have been proposed to explain the electrical variations of these systems as a function of moisture in the ceramic sensors, taking into account the chemisorbed and physisorbed layers of water molecules on the surface of the metal oxide (Figure 1), as well as the capillary condensation of water in the microscopic pores between the particles [7] [8]. Both processes are favored by the existence of a porous structure in the ceramic material.

The changes in the electric response of porous ceramics with different values of ambient humidity are related to the mechanism of adsorption of water on the surface of the oxide. Three conduction regimes can be adopted in these systems: 1) with only a small water coverage of the chemisorbed hydroxyl groups, the hopping conduction of protons is dominant; 2) with a fractional coverage of water (less than one physisorbed layer), diffusion conduction of hydronium in the hydroxyl groups stands out; and 3) when water is abundant, the proton transfer process (Figure 2) is the dominant one.

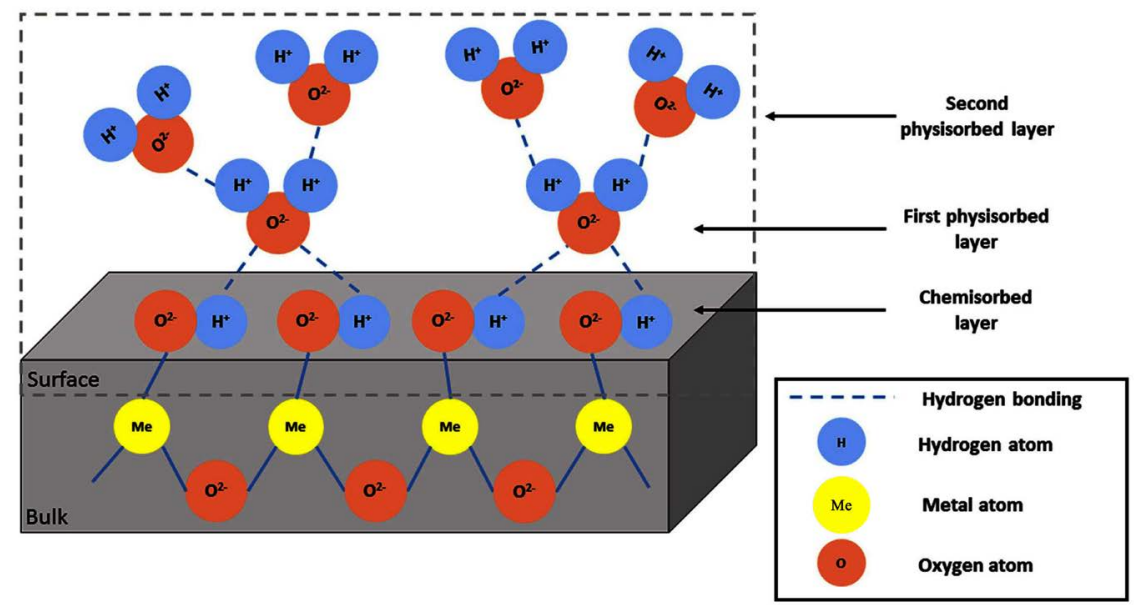

Figure 1. Chemisorbed and physisorbed layers of water molecules formed on the surface of the metal oxide particles with the increase of ambient relative humidity.

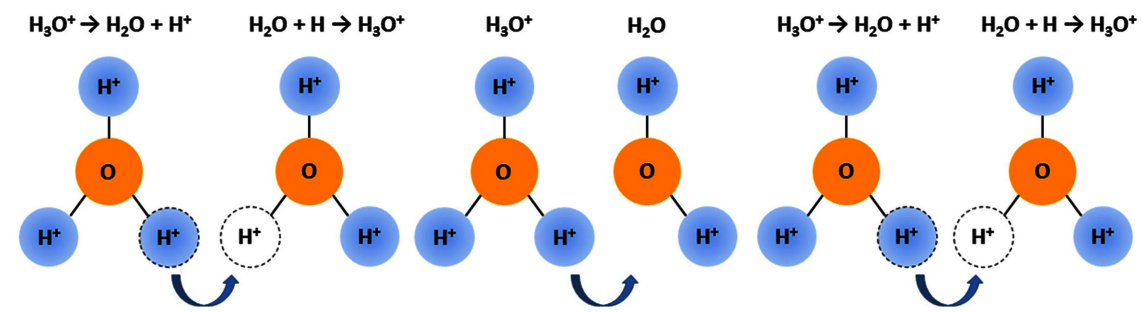

Figure 2. Proton transfer process in the water physisorbed layers on the surface of the metal oxides. 
In this way, the metal oxide sensors can be treated as semiconductor materials, since the electric conduction occurs through moving electrons and electronic holes (load carriers) from one grain to the next, overcoming the potential barrier between the grains [7] [8]. In this context, the electrical impedance spectroscopy technique stands out as one of the most used electric characterization methods in ceramic structures [7] [8]. Similar to resistance, the impedance (Z) is also related to the impediment to the circulation of electric current in a circuit. In a DC (DC) circuit, the resistor is used as the only parameter associated with the electrical response of the system. In the case of an alternating current (AC) circuit, this notion needs to be extended because, in addition to resistors, the so-called inductors and capacitors can be present in the circuit as resistance elements to the passage of an alternating current. To these new resistances, we call reactive resistances or reactances. The main difference between resistance and reactance is in energy storage: in the first case, the energy dissipation is in the form of heat while for the reactance the energy is stored in electric and magnetic fields. In addition, it is still possible to disturb a sample by performing an impedance analysis over a wide range of frequencies, since the potential obeys a sin wave [7] [8].

As expected, a ceramic sensor exhibits impedance variations when subjected to a given relative humidity. The choice of oxides, grain size, distribution and shape of the pores, presence of selective dopants, and fabrication methods are decisive factors to produce devices with greater sensitivity. A moisture sensor of this type may be more efficient (obtain a high sensitivity to moisture) as more porous is its microstructure, the more regular its pore size distribution and the more reactive its surface with water (greater surface area of action) [1] [7] [8]. In other words, advances in nanotechnology have made possible producing ever smaller structures (from the order of the micrometer to the nanometer) with greater surface area/volume ratio and better electrical response for several applications, such as in humidity sensing.

One of the most commonly used metal oxides in moisture sensors is titanium dioxide $\left(\mathrm{TiO}_{2}\right)$ ( $\sim 3.2 \mathrm{eV}$ bandgap), in the allotropic anatase form. This oxide is non-toxic, has high chemical stability and an excellent combination of photoactivity and photostability. Other metal oxides such as tungsten trioxide $\left(\mathrm{WO}_{3}\right)$, zinc oxide $(\mathrm{ZnO})$, vanadium pentoxide $\left(\mathrm{V}_{2} \mathrm{O}_{5}\right)$ and niobium pentoxide $\left(\mathrm{Nb}_{2} \mathrm{O}_{5}\right)$ have been incorporated into $\mathrm{TiO}_{2}$ to form heterogeneous metal oxide materials and increase the efficiency of the sensors [8] [9].

In this context, these heterogeneous metal oxide nanostructures (produced from the sintering of mixtures of these oxides) are potential candidates for development of nanometric scale materials that respond physically and chemically to a given applied stimulus (for example, moisture and light). In addition, numerous and interesting properties have been described in the literature for this class of materials such as better mechanical, thermal, and electronic properties.

One way to improve the efficiency of these moisture sensors and reduce costs 
with material is to produce them to further increase the ratio of surface area of action/volume from the dispersion of these heterogeneous oxides in polymer nanofibers produced by the electrospinning technique (Figure 3), and subsequent sintering of the resulting material [10] [11] [12] [13].

Electrospinning is a simple and interesting method for the synthesis of metal oxide nanofibers [14] [15]. Such structures are produced from an applied electric force (on the order of $\mathrm{kV}$ ) at the tip of a metallic capillary containing a polymer solution and the oxides of interest (or their precursors). This configuration induces the deformation of the drop according to a Taylor's Cone at the tip of the capillary, with consequent formation of metal oxide fibers (with solvent evaporation) toward a metallic collector. The surface area of the nanofibers is maximized compared to fibers of larger diameters, resulting in much greater surface area/volume ratio than the ratio usually obtained. This characteristic directly influences the humidity detection, since there is a greater interaction of particles and substances incorporated in these fibers with the action medium [15].

Based on this, this work presents basic concepts that define the class of semiconductor materials and their functional heteronanostructures based on titanium dioxide $\left(\mathrm{TiO}_{2}\right)$, tungsten trioxide $\left(\mathrm{WO}_{3}\right)$ and selective dopants produced by electrospinning for potential application as moisture sensors.

\section{Fundamentals of Metal Oxide Semiconductors}

Ceramic materials belong to the class of non-metallic inorganic materials, composed of metallic (or semi-metallic) and non-metallic elements bound by ionic and/or covalent bonds, with long-range crystal ordering. Its properties are dependent on the crystalline structure, chemical composition and morphology. Ceramics have good flow resistance, high chemical and physical stability, high temperature resistance, high compressive strength, high hardness and high melt temperature, which give them significant technological applications. Ceramic materials can be classified as ceramic silicates, non-oxide ceramics and ceramic oxides (metal oxides) [16].

Metal oxides have chemical structures with oxygen and metallic elements. These elements are formed by the exchange of charge between highly electronegative oxygen atoms and metal atoms (less electronegative). Metal oxides are widely used to produce gas sensors because of their excellent thermal and environmental stability, as well as good reversibility of the electric response. In addition, the surface of the metal oxides shows a fundamental role in the application of these materials in flexible electronics. For example, the good performance of these ceramic oxides as catalysts and moisture sensors is directly related to the properties of their surfaces or the interfaces between these oxides and the action medium/other materials. In addition, the metal oxides exhibit unique optical, optoelectronic, magnetic, electrical, mechanical, thermal, catalytic and photochemical characteristics, which give it a series of applications in flexible electronics such as in fuel cells, solar cells, and sensors [17]-[23]. 


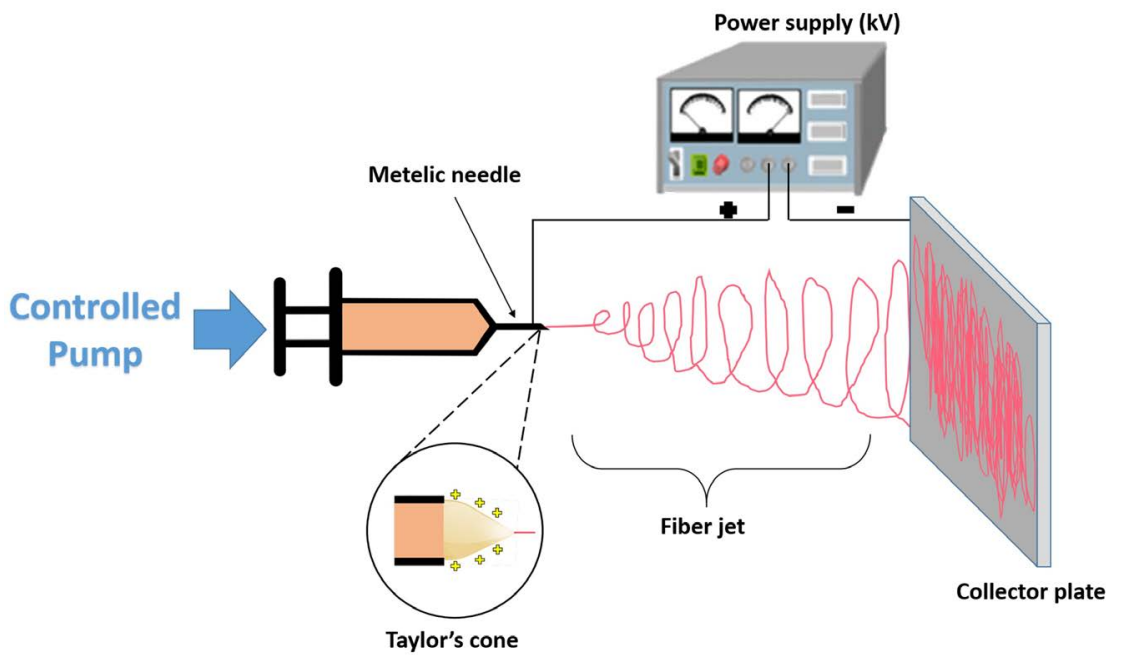

Figure 3. Electrospinning process for the production of nanofibers.

These materials can be produced through various synthesis methods, when the metal elements lose electrons to the oxygen atom to form oxides with different bonds and electronic structures, which gives them electrical properties characteristic of a semiconductor. Semiconductor materials have intermediate electrical behavior between conductors and insulators, and can behave in one way or another by controlling the supply of stimulus (heat, light, electric field, pressure, etc.) and/or changes in their composition and morphology [24]. The preparation with various morphologies, the possibility of obtaining metal oxide nanostructures of controlled size, shape and composition and interaction with other structures, as well as the possibility of molding and dispersing them in other materials increase the attributes of these materials and open new perspectives for technological applications in the field of electronics.

Semiconductors can be intrinsic (pure) or extrinsic (when other substances are added to change their properties). The synthesis process of extrinsic semiconductors by the addition (doping) of small amounts of impurities in a pure semiconductor, for the purpose of modulating its electrical properties, may improve the conductivity of the material. Semiconductors are doped to get a smaller band gap by introducing energetic levels in the forbidden band generated by excess of electrons (electron donor elements form n-type semiconductors) or an electron deficiency (electron acceptor elements form p-type semiconductors) [18]-[25].

Electron deficiency creates electronic holes that, like electrons, are charge carriers responsible for creating electrical current in semiconductors. While an electron has a negative charge, the holes have opposite polarity (positive charge) to the charge of the electron. The electrons motion of the valence band to the conduction band also generates holes in the origin band, with consequent increase of the electron deficiency. Both electrons and holes are vital for creating current in semiconductors. When applying an external electrical potential difference, both electrons and holes can have mobility between the conduction and 
valence bands. This behavior is responsible for the semiconductor performance and for many of the electrical and thermal properties of the solids, forming the basis of the solid state electronics technology [18]-[25].

When the particle size of the semiconductor metal oxides is on the nanometer scale (of the order of $10^{-9} \mathrm{~m}$ ), their physical and chemical properties described above may be improved. The specific surface area (as well as the surface area/volume ratio) increase significantly when the dimensions of the material size decrease. The movement of electrons and holes in semiconductor nanomaterials are also affected by the geometry of the particles, since smaller particles have the highest density of surface defects, promote the reduction of the free path between the particles and increase both the number of collisions experienced by the charge carriers as to the strength of the material [20] [26] [27] [28] [29]. This class of nanostructured materials is considered one of the most functional and therefore a topic of active research today. All these properties make them highly desirable materials in emerging applications such as gas sensors, especially in humidity sensors. Among the many approaches, sensors based on composite metal oxides have been extensively investigated, since these semiconductors can have conduction mechanisms that are highly influenced by their improved physical/chemical properties [14].

The selective adsorption of water vapor, the activation of the transport mechanisms, the microstructure and the number of water adsorption sites may be modified by the addition of suitable contaminants to the sensor ceramic materials or by modifications of their chemical composition. The change of these parameters promotes changes in the impedance (resistance to the passage of electric current) that the semiconductor material experiences when exposed to a certain concentration of humidity, which may qualify it as a relative humidity (RH) sensor. In these cases, the sensitivity of the material to $\mathrm{RH}$ is influenced by the porous microstructure (and consequently the greater surface area) and the reactivity of its surface with RH. Therefore, if the sensing capacity of these metal oxides depends on the microstructure and composition, the control of these parameters in the detection material is a strategic way to obtain a better response of the sensors across the UR range [30].

The electron/ionic charge transfer reactions occurring on the surface of the semiconductor and in the pores between the particles can be used to explain the detection of moisture. The mechanism of conduction of the humidity sensors can be of the ionic, electronic or mixed type (when it presents contributions of the two mechanisms). In addition, heterogeneous semiconductor materials, produced from the interaction of the mixture between two or more oxides, may have greater activity, selectivity and stability and, therefore, have attracted a lot of attention in recent years [31].

\section{Potential Metal Oxide Heteronanostructures for Humidity Detection}

Recent works by Chen et al., Blank et al. and Farahani et al. discussed the elec- 
trical behavior of several semiconductor oxides. In addition, many other aspects related to moisture detection such as alternative methods of synthesis and the improvement of the microstructure of the sensors from the use of dopant or hybrid compositions have also been discussed [30] [32] [33].

As stated, the electrical response of porous ceramics to changes in moisture concentration is strongly related to the adsorption mechanisms of water on the surface of the oxide and in the pores [7] [33]. Factors such as particle size distribution, grain orientation, grain boundary, strain-strain and atomic defect distribution may influence the electrical behavior of a material [34] [35] [36]. Thus, doping a composite material with additional semiconductor metal oxides will induce additional atomic defects with consequent changes in the electrical response of the sensor. The effect of dopants is related to their ion charge/volume ratio and their concentration on the surface of the resulting material, which increases the number of adsorption sites for the water molecules. Proper choice of dopants can improve sensitivity, linearity of signal response, stability, and performance of sensing devices.

For example, Nenov et al. investigated ceramic elements of moisture detection based on $\mathrm{TiO}_{2}$ with dopants such as $\mathrm{PbO}$ and $\mathrm{Bi}_{2} \mathrm{O}_{3}$ : the influence of doping and sintering temperature on the characteristics of ceramic moisture sensors was studied and experimental samples based on the optimal compositions and sintering temperatures were extensively investigated [37]. Thus, following a similar strategy, our research group has proposed to analyze the electrical response of nanostructures of metal oxides based on $\mathrm{TiO}_{2}$ and $\mathrm{WO}_{3}$ (and doped with other selective oxides) submitted to moist atmospheres.

The technological attraction for the use of $\mathrm{TiO}_{2}$ and $\mathrm{WO}_{3}$ nanostructures (and their mixtures with other oxides) for humidity detection is based on the fact that these materials possess, in addition to excellent chemical/structural stability, high surface area/volume ratio (compared to usual ceramic structures used for the same end) combined with numerous electrical properties such as piezoelectricity, pyroelectricity, superconductivity, ferroelectricity, among others. Due to these punctual characteristics, the use of these nanostructures has been growing strongly in recent years as an open field of research both for synthesis and characterization as for the structural improvement of these materials for the industry [10] [11].

Amongst numerous semiconductor oxides, $\mathrm{TiO}_{2}$ and $\mathrm{WO}_{3}$ deserve special attention in the production of heteronanostructures as they are technologically important materials due to their excellent electronic, optical, chemical and structural properties. When combined from sintering processes, these oxides may form heterogeneous ceramic nanostructures with lower bandgap, which favors the conduction processes in these materials for the application as sensors. In addition, the heterogeneous material presents higher conductivity in relation to the pure $\mathrm{TiO}_{2}$ (ratio of 1:1 in mol) [8] [9] [10] [11].

Besides that, dopants such as $\mathrm{Nb}_{2} \mathrm{O}_{5}, \mathrm{~V}_{2} \mathrm{O}_{5}, \mathrm{CuO}$ and $\mathrm{ZnO}$ present similar io- 
nic radius to $\mathrm{TiO}_{2}$ and may be incorporated into their electronic structure after sintering process, in an attempt to retard the thermally activated transition from anatase (preferential) to rutile. In addition to these specific characteristics, all the oxides studied are highly sensitive to variations in moisture. These characteristics are essential to produce ceramic sensors with high sensitivity. These materials have been tested as moisture sensors from a 1:1 molar ratio mixture between $\mathrm{TiO}_{2}$ and $\mathrm{WO}_{3}$ (and with selective dopants), as pellets $(8 \mathrm{~mm} \times 6 \mathrm{~mm} \times 1$ $\mathrm{mm}$ ), and showed excellent sensitivity to variations of relative humidity [8] [9].

Recently, these ceramic nanostructures were also prepared from the electrospinning of an organic matrix containing the mixture of the metal oxides, combined with the sintering process. The novel preparation procedure (Figure 4) also showed potential for the development of nanostructured ceramic sensors, since the resulting materials presented high porosity and surface area/volume ratio (compared to the usual ceramic sensors produced in the form of volumetric pellets), offering a greater interaction of the particles and incorporated substances with the action medium.

With this novel preparation route it is possible to produce thin films of the material instead of volumetric pellets, using a much smaller amount of material than the one used to produce pellets, without loss of sensorial quality and with lower cost. It is also possible to produce metal oxides nanofibers from the electrospinning of their precursors. It is also possible to control the diameter of the fibers by varying the parameters of the precursor solution and the potential difference applied at the tip of the metallic capillary containing this solution.

As discussed, the route of preparation of the nanostructures follows the steps shown in Figure 4:1) $\mathrm{TiO}_{2}: \mathrm{WO}_{3}$ oxides are encapsulated in polymeric nanofibers by electrospinning (Figure 4(a) and Figure 4(b)); 2) $\left(\mathrm{TiO}_{2}: \mathrm{WO}_{3}\right)$-loaded fibers layers are arranged on electrodes (Figure $4(\mathrm{c}) ; 3$ ) the polymer-ceramic composite is then sintered $\left(\right.$ at $500^{\circ} \mathrm{C}, 700^{\circ} \mathrm{C}$, etc.) to eliminate the polymer phase

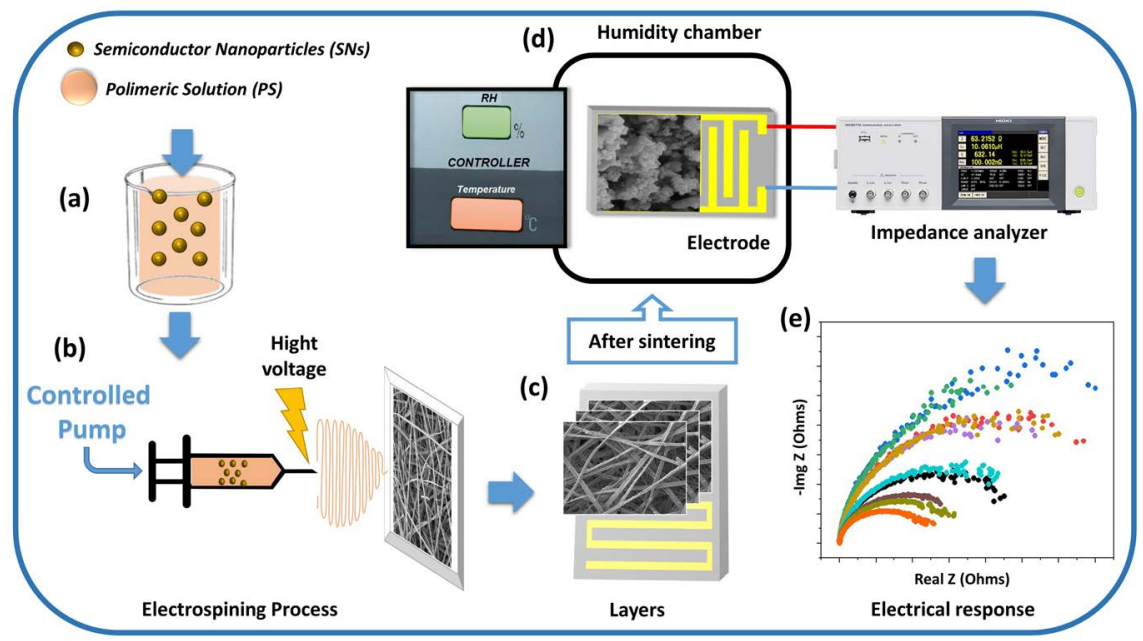

Figure 4. Step by step for the preparation of the heteronanostructures by electrospinning and sintering. 
and to promote the regular dispersion of the oxides (free of polymer) on the electrode surface (Figure 4(d)) for moisture detection tests in a humidity chamber. The electrospinning process parameters such as working temperature, sintering temperature and number of fibers layers can also be analyzed in order to optimize the electrical response of the materials to the variations of humidity [13].

In addition, the choice of the electrospinning technique for the production of high surface area nanometric ceramics is promising for the application as sensors because it presents some advantages such as flexibility in the choice of materials and design, the possibility of molding the fibrous material on other surfaces, low cost in automation, mass production and good reproducibility of results [15].

\section{Conclusion}

Basic concepts of metallic oxides were presented. Electrical/electronic properties that define the conduction mechanisms of these materials were discussed as a function of ambient relative humidity. In addition, it has shown the possibility of producing heteronanostructures based on $\mathrm{TiO}_{2}, \mathrm{WO}_{3}$ and their selective dopants from the alternative preparation route by electrospinning and sintering. It is expected that this work will contribute to future research involving the use of semiconductor oxides heteronanostructures as humidity sensors.

\section{Acknowledgements}

The authors acknowledge the financial support from Bahia State Research Foundation (FAPESB, Project 1252/2018).

\section{Conflicts of Interest}

The authors declare no conflicts of interest regarding the publication of this paper.

\section{References}

[1] Faia, P.M., Furtado, C.S. and Ferreira, A.J. (2004) Humidity Sensing Properties of a Thick-Film Titania Prepared by a Slow Spinning Process. Sensors and Actuators B: Chemical, 101, 183-190. https://doi.org/10.1016/j.snb.2004.02.050

[2] Wang, M.Y., Zhang, D.Z., Yang, A.J., Wang, D.R. and Zong, X.Q. (2019) Fabrication of Polypyrrole/Graphene Oxide Hybrid Nanocomposite for Ultrasensitive Humidity Sensing with Unprecedented Sensitivity. Journal of Materials Science Materials in Electronics, 30, 4967-4976. https://doi.org/10.1007/s10854-019-00793-4

[3] Zhang, D.Z., Zong, X.Q. and Wu, Z.L. (2019) Fabrication of Tin Disulfide/Graphene Oxide Nanoflower on Flexible Substrate for Ultrasensitive Humidity Sensing with Ultralow Hysteresis and Good Reversibility. Sensors and Actuators B: Chemical, 287, 398-407. https://doi.org/10.1016/j.snb.2019.01.123

[4] Zhang, D., Sun, Y., Li, P. and Zhang, Y. (2016) Facile Fabrication of MoS2-Modified $\mathrm{SnO}_{2}$ Hybrid Nanocomposite for Ultrasensitive Humidity Sensing. ACS Applied Materials \& Interfaces, 8, 14142-14149. https://doi.org/10.1021/acsami.6b02206 
[5] Zhang, D., Liu, J. and Xia, B. (2016) Layer-by-Layer Self-Assembly of Zinc Oxide/Graphene Oxide Hybrid toward Ultrasensitive Humidity Sensing. IEEE Electron Device Letters, 37, 916-919. https://doi.org/10.1109/LED.2016.2565728

[6] Zhang, D., Chang, H., Li, P., Liu, R. and Xue, Q. (2016) Fabrication and Characterization of an Ultrasensitive Humidity Sensor Based on Metal Oxide/Graphene Hybrid Nanocomposite. Sensors and Actuators B: Chemical, 225, 233-240. https://doi.org/10.1016/j.snb.2015.11.024

[7] Traversa, E. (1995) Ceramic Sensors for Humidity Detection: The State-of-the-Art and Future Developments. Sensors and Actuators B: Chemical, 23, 135-156. https://doi.org/10.1016/0925-4005(94)01268-M

[8] Faia, P.M., Jesus, E.L. and Louro, C.S. (2014) $\mathrm{TiO}_{2}: \mathrm{WO}_{3}$ Composite Humidity Sensors Doped with $\mathrm{ZnO}$ and $\mathrm{CuO}$ Investigated by Impedance Spectroscopy. Sensors and Actuators B: Chemical, 203, 340-348. https://doi.org/10.1016/j.snb.2014.06.117

[9] Faia, P.M., Libardi, J. and Louro, C.S. (2016) Effect of $\mathrm{V}_{2} \mathrm{O}_{5}$ Doping on p- to n-Conduction Type Transition of $\mathrm{TiO}_{2}: \mathrm{WO}_{3}$ Composite Humidity Sensors. Sensors and Actuators B: Chemical, 222, 952-964. https://doi.org/10.1016/j.snb.2015.09.039

[10] Araújo, E.S., Nascimento, M.L.F. and de Oliveira, H.P. (2013) Influence of Triton X-100 on PVA Fibres Production by the Electrospinning Technique. Fibres \& Textiles in Eastern Europe, 21, 39-43.

[11] Araújo, E.S., Libardi, J., Faia, P.M. and de Oliveira, H.P. (2017) Humidity-Sensing Properties of Hierarchical $\mathrm{TiO}_{2}: \mathrm{ZnO}$ Composite Grown on Electrospun Fibers. Journal of Materials Science: Materials in Electronics, 28, 16575-16583.

https://doi.org/10.1007/s10854-017-7571-5

[12] Araújo, E.S., Libardi, J., Faia, P.M. and de Oliveira, H.P. (2018) Characterization and Electrical Response to Humidity of Sintered Polymeric Electrospun Fibers of Vanadium Oxide- $\mathrm{TiO}_{2} / \mathrm{WO}_{3}$. Journal of Electronic Materials, 47, 2710-2717. https://doi.org/10.1007/s11664-018-6112-1

[13] Araújo, E.S. and Leão, V.N.S. (2019) $\mathrm{TiO}_{2} / \mathrm{WO}_{3}$ Heterogeneous Structures Prepared by Electrospinning and Sintering Steps: Characterization and Analysis of the Impedance Variation to Humidity. Journal of Advanced Ceramics, 8, 238-246. https://doi.org/10.1007/s40145-018-0309-x

[14] Araujo, E.S., Libardi, J., Faia, P.M. and de Oliveira, H.P. (2015) Hybrid $\mathrm{ZnO} / \mathrm{TiO}_{2}$ Loaded in Electrospun Polymeric Fibers as Photocatalyst. Journal of Chemistry, 2015, Article ID: 476472. https://doi.org/10.1155/2015/476472

[15] Huang, Z.M., Zhang, Y.Z., Kotaki, M. and Ramakrishna, S. (2003) A Review on Polymer Nanofibers by Electrospinning and Their Applications in Nanocomposites. Composites Science and Technology, 63, 2223-2253. https://doi.org/10.1016/S0266-3538(03)00178-7

[16] Sudha, P.N., Sangeetha, K., Jisha Kumari, A.V., Vanisri, N. and Rani, K. (2018) Corrosion of Ceramic Materials. In: Thomas, S., Balakrishnan, P. and Sreekala, M.S., Eds., Fundamental Biomaterials. Ceramics, Woodhead Publishing, Soston, 223-250.

[17] Hoffmann, M.R., Martin, S.T., Choi, W.Y. and Bahnemann, D.W. (1995) Environmental Applications of Semiconductor Photocatalysis. Chemical Reviews, 95, 69-96. https://doi.org/10.1021/cr00033a004

[18] Trasatti, S. (1991) Physical Electrochemistry of Ceramic Oxides. Electrochimica Acta, 36, 225-241. https://doi.org/10.1016/0013-4686(91)85244-2

[19] Tong, H., Ouyang, S.X., Bi, Y.P., Umezawa, N., Oshikiri, M. and Ye, J.H. (2012) Nano-Photocatalytic Materials: Possibilities and Challenges. Advanced Materials, 24, 229-251. https://doi.org/10.1002/adma.201102752 
[20] Dey, A. (2018) Semiconductor Metal Oxide Gas Sensors: A Review. Materials Science and Engineering B-Advanced Functional Solid-State Materials, 229, 206-217. https://doi.org/10.1016/j.mseb.2017.12.036

[21] Chen, X. and Mao, S.S. (2007) Titanium Dioxide Nanomaterials: Synthesis, Properties, Modifications, and Applications. Chemical Reviews, 107, 2891-2959. https://doi.org/10.1021/cr0500535

[22] Comini, E., Baratto, C., Faglia, G., Ferroni, M., Vomiero, A. and Sberveglieri, G. (2009) Quasi-One Dimensional Metal Oxide Semiconductors: Preparation, Characterization and Application as Chemical Sensors. Progress in Materials Science, 54, 1-67. https://doi.org/10.1016/j.pmatsci.2008.06.003

[23] Diao, F.Y. and Wang, Y.Q. (2018) Transition Metal Oxide Nanostructures: Premeditated Fabrication and Applications in Electronic and Photonic Devices. Journal of Materials Science, 53, 4334-4359. https://doi.org/10.1007/s10853-017-1862-3

[24] Batzill, M. and Diebold, U. (2005) The Surface and Materials Science of Tin Oxide. Progress in Surface Science, 79, 47-154. https://doi.org/10.1016/j.progsurf.2005.09.002

[25] Hu, C. (2010) Modern Semiconductor Devices for Integrated Circuits. Prentice Hall, Upper Saddle River.

[26] Barsan, N., Schweizer-Berberich, M. and Gopel, W. (1999) Fundamental and Practical Aspects in the Design of Nanoscaled $\mathrm{SnO}_{2}$ Gas Sensors: A Status Report. Fresenius Journal of Analytical Chemistry, 365, 287-304. https://doi.org/10.1007/s002160051490

[27] Gurlo, A., Ivanovskaya, M., Barsan, N., Schweizer-Berberich, M., Weimar, U., Gopel, W., et al. (1997) Grain Size Control in Nanocrystalline $\operatorname{In}_{2} \mathrm{O}_{3}$ Semiconductor Gas Sensors. Sensors and Actuators B: Chemical, 44, 327-333. https://doi.org/10.1016/S0925-4005(97)00199-8

[28] El-Sayed, M.A. (2001) Some Interesting Properties of Metals Confined in Time and Nanometer Space of Different Shapes. Accounts of Chemical Research, 34, 257-264. https://doi.org/10.1021/ar960016n

[29] Franke, M.E., Koplin, T.J. and Simon, U. (2006) Metal and Metal Oxide Nanoparticles in Chemiresistors: Does the Nanoscale Matter? Small, 2, 36-50. https://doi.org/10.1002/smll.200500261

[30] Farahani, H., Wagiran, R. and Hamidon, M.N. (2014) Humidity Sensors Principle, Mechanism, and Fabrication Technologies: A Comprehensive Review. Sensors, 14, 7881-7939. https://doi.org/10.3390/s140507881

[31] Faia, P.M. and Furtado, C.S. (2013) Effect of Composition on Electrical Response to Humidity of $\mathrm{TiO}_{2}: \mathrm{ZnO}$ Sensors Investigated by Impedance Spectroscopy. Sensors and Actuators B: Chemical, 181, 720-729. https://doi.org/10.1016/j.snb.2013.02.027

[32] Chen, Z. and Lu, C. (2005) Humidity Sensors: A Review of Materials and Mechanisms. Sensor Letters, 3, 274-295. https://doi.org/10.1166/sl.2005.045

[33] Blank, T.A., Eksperiandova, L.P. and Belikov, K.N. (2016) Recent Trends of Ceramic Humidity Sensors Development: A Review. Sensors and Actuators B: Chemical, 228, 416-442. https://doi.org/10.1016/j.snb.2016.01.015

[34] Chou, K.S., Lee, T.K. and Liu, F.J. (1999) Sensing Mechanism of a Porous Ceramic as Humidity Sensor. Sensors and Actuators B: Chemical, 56, 106-111. https://doi.org/10.1016/S0925-4005(99)00187-2

[35] Tripathy, A., Pramanik, S., Manna, A., Radzi, Z. and Abu Osman, N.A. (2017) Dielectric and AC Conductivity Studies of Novel Porous Armalcolite Nanocomposite-Based 
Humidity Sensor. Journal of the American Ceramic Society, 100, 5131-5140. https://doi.org/10.1111/jace.15033

[36] Saha, D. and Das, S. (2018) Effect of Porosity on the Response of Alumina Thick Films towards Moisture. Transactions of the Indian Ceramic Society, 77, 138-145. https://doi.org/10.1080/0371750X.2018.1502052

[37] Nenov, T. and Nenova, Z. (2013) Multi-Objective Optimization of the Parameters of $\mathrm{TiO}_{2}$-Based Ceramic Humidity Sensors. Ceramics International, 39, 4465-4473. https://doi.org/10.1016/j.ceramint.2012.11.040 